\title{
Antiphospholipid antibodies in patient with acute lower member ischemia and pulmonary thromboembolism as a result of infection by SARS-CoV2
}

\author{
C. Sieiro Santos ${ }^{1} \cdot$ C. Nogal Arias ${ }^{2} \cdot$ C. Moriano Morales ${ }^{1} \cdot$ M. Ballesteros Pomar ${ }^{2} \cdot$ E. Diez Alvarez ${ }^{1} \cdot$ T. Perez Sandoval $^{1}$
}

Received: 11 May 2020 / Revised: 11 May 2020 / Accepted: 19 May 2020 / Published online: 4 June 2020

(C) International League of Associations for Rheumatology (ILAR) 2020

Dear Sirs,

SARS-CoV-2 infection has spread to more than 140 countries, according to the WHO. More than $80 \%$ of cases of SARS-CoV-2 infection have flu-like symptoms, $20 \%$ require hospitalization and $5-15 \%$ intensive care [1]. Hospitalized patients tend to die from symptoms of acute respiratory distress syndrome and, in some cases, multi-organ failure [2]. In critically ill patients, there are laboratory abnormalities that suggest a cytokine storm in response to viral infection leading to uncontrolled proliferation of T cells, excessive macrophage activation and hypersecretion of proinflammatory cytokines, IL-1 $\beta$, IL-6, interferon and tumoural necrosis factor $\alpha$ $(\mathrm{TNF} \alpha)$ [3]. The activation of thrombin is also found in these patients, with multiple thrombotic events ranging from peripheral ischemia, pulmonary thromboembolism to disseminated intravascular coagulation (DIC). These complications were the cause of death in many of these patients. Thrombocytopenia and increased D-dimer levels, as well as prolongation of the prothrombin time (PT) and international normalized ratio (INR), are associated with a higher disease severity [4].

Recently, the New England Journal of Medicine published a series of cases related to infection by SARS-CoV2 and bilateral limb ischemia and elevated antiphospholipid antibodies. It is not clear whether antiphospholipid antibodies play a

C. Sieiro Santos and C. Nogal Arias contributed equally to this work.

C. Sieiro Santos

cristysieirosantos@gmail.com

1 Rheumatology Department, Complejo Asistencial Universitario de León, Leon, Spain

2 Cardiovascular Surgery Department, Complejo Asistencial Universitario de León, Leon, Spain major role in the pathophysiology of thrombosis associated with COVID-19 [5]. Here we describe a patient with arterial and venous ischemia as a result of infection by SARS-CoV2 that was positive for antiphospholipid antibodies after discharge. A 70-year-old patient with hypertension and diabetes presented to the urgency room with symptoms of ischemia in lower members. The patient had no history of thrombotic events, abortions or rheumatic diseases. The patient had fever and respiratory symptoms 1 week prior coming to the urgency room but at the moment of examination had no symptoms of dyspnoea, diarrhoea, cough or headache. The patient had a temperature of $36.5^{\circ}$, and basal oxygen saturation was $98 \%$. On examination, patient had signs of coldness, loss of sensibility and motor skills in the right leg which suggest acute ischemia. Pulmonary auscultation revealed crackles. Cardiac auscultation was normal. A chest X-ray showed a reticularnodular pattern with peripheral distribution in lower lung bases. A CT angiography revealed an acute pulmonary thromboembolism affecting the apical segmental artery (right inferior lobe) and posterior segmental artery (left inferior lobe). The lung parenchyma showed multiple patched areas of increased attenuation in frosted glass and peripheral distribution, in both lung fields, and typical "crazy paving" pattern, mainly in the posterior/lateral segment of the right and left inferior lobes, lateral segment of the medium lobe and lingula. Signs of acute thrombi in the abdominal aorta and right common iliac and obstruction of the second portion of right popliteal were also found. All these findings were consistent with a typical pattern of COVID-19 infection. Reverse transcriptase-polymerase chain reaction of nasopharyngeal and sputum swabs was negative; however, the presence of IgG antibodies against SARS-CoV2 was detected which suggested infection by COVID-19. Venous blood gases showed a pH of 7.28, $\mathrm{pCO}_{2}$ of 38,2 and $\mathrm{HCO}_{3}$ of 17 which was consisted with acidosis. Laboratory tests are depicted in Table 1, with signs of renal failure (creatinine 
Table 1 Laboratory tests

Laboratory findings

\begin{tabular}{ll}
\hline White cell count $\left(\mathrm{mm}^{3}\right)$ & 28.800 \\
Neutrophils $\left(\mathrm{mm}^{3}\right)$ & 81.000 \\
Lymphocytes $\left(\mathrm{mm}^{3}\right)$ & 9.000 \\
Platelet count $\left(\mathrm{mm}^{3}\right)$ & 382.000 \\
Haemoglobin $\left(\mathrm{mm}^{6}\right)$ & 12,3 \\
INR & 1,32 \\
Alanine aminotransferase (U/l) & 231 \\
Aspartate aminotransferase $(\mathrm{U} / \mathrm{l})$ & 149 \\
Lactate dehydrogenase (U/l) & 668 \\
Creatinine $(\mu \mathrm{mol} / \mathrm{l})$ & 2,38 \\
Creatine kinase $(\mathrm{U} / \mathrm{l})$ & 11.427 \\
Creatine kinase $\mathrm{MB}$ isoenzyme (U/l) & 311 \\
EGFR (ml/min/1.73 m $\left.{ }^{2}\right)$ & 20 \\
Cardiac troponin I (pg/ml) & 17.83 \\
Prothrombin time $(\mathrm{s})$ & 15,2 \\
Activated partial thromboplastin time (s) & 55 \\
Fibrinogen (g/l) & 584 \\
D-dimer (mg/l) & 71.016 \\
Serum ferritin (ng/ml) & 623 \\
Procalcitonin (ng/ml) & 0,2 \\
High-sensitivity C-reactive protein (mg/l) & 100,5 \\
Pro-BNP & 761,2 \\
Ions & Sodium 135 mmol/1 \\
Antiphospholipid antibodies & Potassium 5,.8 mmol/1 \\
& Positive for lupus \\
& anticoagulant, positive \\
\hline & for IgG cardiolipin \\
\hline
\end{tabular}

2,38, urea 163 and glomerular filtrate of 20) and high levels of transaminases (ALT 231, ASPT 149), LDH 669, CK 11.427 and D-dimer 72,016. Initial treatment with empiric antibiotic therapy, hydroxychloroquine and lopinavir/ritonavir was implemented. Due to high thrombotic risk, the patient received treatment with low-molecular-weight heparin at therapeutic dose and needed percutaneous thrombectomy for acute popliteal obstruction. Treatment with intensive fluid therapy and bicarbonate was also required in order to improve renal function. After discharge, the patient was tested twice for antiphospholipid antibodies and was positive for anticardiolipin IgG antibodies as well as lupus anticoagulant.

This report emphasizes that thrombotic disease may have precedent factors or incident complications in patients with COVID-19 and that antiphospholipid antibodies may play a role in the pathophysiology of thrombosis; however, more studies are required to determine whether there is an association.

Acknowledgements The authors acknowledge the assistance of study participant, radiographers, study nurses and laboratory staff who participated in the study.

\section{Compliance with ethical standards}

Patient consent was given with purpose of writing this article. Disclosures None.

\section{References}

1. Pan F, Ye T, Sun P, Gui S, Liang B, Li L, Zheng D, Wang J, Hesketh RL, Yang L, Zheng C (2020:200370) Time course of lung changes on chest CT during recovery from 2019 novel coronavirus (COVID19) pneumonia. Radiology. 295:715-721

2. Wang D, Hu B, Hu C, Zhu F, Liu X, Zhang J, Wang B, Xiang H, Cheng Z, Xiong Y, Zhao Y, Li Y, Wang X, Peng Z (2020) Clinical characteristics of 138 hospitalized patients with 2019 novel coronavirus-infected pneumonia in Wuhan, China. JAMA 323:1061

3. Mehta P, McAuley DF, Brown M, Sanchez E, Tattersall RS, Manson JJ (2020) COVID-19: consider cytokine storm syndromes and immunosuppression. Lancet. 395:1033-1034

4. Tang N, Li D, Wang X, Sun Z (2020) Abnormal coagulation parameters are associated with poor prognosis in patients with novel coronavirus pneumonia. J Thromb Haemost 18:844-847

5. Zhang Y, Xiao M, Zhang S (2020) Coagulopathy and antiphospholipid antibodies in patients with Covid-19. NEJM 382: e38

Publisher's note Springer Nature remains neutral with regard to jurisdictional claims in published maps and institutional affiliations. 